\title{
Association between AXIN1 Gene Polymorphisms and Bladder Cancer in Chinese Han Population
}

\author{
Qin Li $\mathbb{D}^{1,2}$ Peng Zhang, ${ }^{3}$ Yanyun Wang $\mathbb{D},{ }^{1}$ Yan Zhang, ${ }^{1,4}$ Kai Li, ${ }^{1,5}$ Yaping Song, ${ }^{1}$ Min Su, \\ Bin Zhou $\mathbb{D}^{1},{ }^{1}$ and Lin Zhang $\mathbb{D}^{1,2}$ \\ ${ }^{1}$ Laboratory of Molecular Translational Medicine, Center for Translational Medicine, Key Laboratory of Birth Defects and Related \\ Diseases of Women and Children (Sichuan University), Ministry of Education, West China Second University Hospital, \\ Sichuan University, Chengdu, Sichuan 610041, China \\ ${ }^{2}$ Department of Immunology, West China School of Preclinical and Forensic Medicine, Sichuan University, Chengdu, \\ Sichuan 610041, China \\ ${ }^{3}$ Department of Urology, West China Hospital, Sichuan University, Chengdu, Sichuan, China \\ ${ }^{4}$ Department of Pathology, West China Second University Hospital, Sichuan University, Chengdu, Sichuan 610041, China \\ ${ }^{5}$ Department of Cardiology, West China Hospital, Sichuan University, Chengdu, Sichuan 610041, China
}

Correspondence should be addressed to Bin Zhou; zb630@163.com and Lin Zhang; zhanglin@scu.edu.cn

Received 7 August 2018; Revised 28 January 2019; Accepted 7 February 2019; Published 15 April 2019

Academic Editor: Monica Cantile

Copyright (C) 2019 Qin Li et al. This is an open access article distributed under the Creative Commons Attribution License, which permits unrestricted use, distribution, and reproduction in any medium, provided the original work is properly cited.

\begin{abstract}
Background. Previous evidence has indicated that the reduction of axis inhibition protein 1 (AXIN1) expression is related with the poor differentiation of non-small-cell lung cancer (NSCLC). However, the potential association between AXIN1 and bladder cancer (BC) is unknown. We aimed to initially explore the relevance of AXIN1 gene polymorphisms (rs12921862 C/A, rs1805105 T/C, and rs370681 C/T) and BC. Methods. Three hundred and sixteen BC patients and 419 healthy controls had been enrolled. Polymerase chain reaction-restriction fragment length polymorphism (PCR-RFLP) method was used for genotyping three tag single-nucleotide polymorphisms (SNPs) of AXIN1. The SNPstats online analysis software and SPSS software were used for statistical analysis. Results. Our data revealed that three tag SNPs were associated with an increased risk of BC $($ rs12921862: $P<0.001$, OR $(95 \% \mathrm{CI})=4.61(3.13-6.81)$; rs1805105: $P=0.046$, OR $(95 \% \mathrm{CI})=1.35(1.00-1.82)$; and rs370681: $P=0.004$, OR $(95 \% \mathrm{CI})=1.56(1.15-2.10))$. For rs12921862, A allele was an independently protective factor which correlated with a better prognosis in non-muscle-invasive bladder cancer (NMIBC) patients $(P=0.03, \mathrm{OR}(95 \% \mathrm{CI})=0.10$ (0.01-0.84)). Stratification analysis demonstrated that $\mathrm{rs} 370681$ polymorphism was related with high-grade bladder cancer $(P=0.04$, OR $(95 \% \mathrm{CI})=1.85(1.04-3.23))$. Conclusion. The AXIN1 gene polymorphisms might implicate in BC risk, and rs 12921862 could be a potential forecasting factor for prognosis of BC patients.
\end{abstract}

\section{Introduction}

Bladder cancer $(\mathrm{BC})$ was ranked as the ninth most common cancer by the International Agency for Research on Cancer in 2012. It is related to thrice as much higher morbidity and mortality in the developed countries compared to the developing regions, and more than $75 \%$ of the cases occur in men. In 2012, 165000 deaths and 429000 new cases were recorded worldwide, of which 26820 deaths and 55486 new cases were in China, resulting in an incidence rate of $3 / 100000$ and accounting for a large fraction of $\mathrm{BC}$ in East
Asia (37491 deaths and 85451 cases) [1]. Around $80 \%$ of $\mathrm{BC}$ patients have the non-muscle-invasive bladder cancer (NMIBC), whereas muscle-invasive bladder cancers (MIBC) account for only $20 \%$ of the cases, although they are responsible for a large number of deaths [2-4].

Smoking is the most significant risk factor of BC, accounting for an estimated $50 \%$ of all cases, followed by occupational exposure to chemical carcinogens, e.g., industrial paints, dyes, metals, and petroleum products [3-5]. Increasing evidences in recent years have suggested a genetic predisposition towards cancer susceptibility. For instance, 
$\mathrm{Gu}$ et al. found an association between the N-acetyl transferase 2 (NAT2) slow acetylator phenotype and a significantly higher risk of $\mathrm{BC}$ in smokers [6]. Moreover, the risk of $\mathrm{BC}$ is twofold higher in first-degree relatives of BC patients [4], indicating that several genetic factors could play a role in the initiation and progression of BC.

The Wnt/ $\beta$-catenin signaling pathway is one of the fundamental pathways regulating cell proliferation, polarity, and lineage differentiation during embryonic development and tissue homeostasis, and mutations in its components are often involved in birth defects, cancer, and other diseases $[7,8]$. Studies have directly linked the Wnt signaling pathway to the development of $\mathrm{BC}$ [9-11]. Mao et al. demonstrated that activating the Wnt pathway could accelerate the epithelial-mesenchymal transition (EMT), invasion, and migration of $\mathrm{BC}$ cells in vitro [12]. In addition, the inhibition of Wnt signaling suppressed BC xenograft growth in nude mice [13]. Canonical Wnt signaling is regulated by the degradation of the AXIN1-mediated $\beta$-catenin destruction complex. Axis inhibition protein 1 (AXIN1) is a multidomain scaffold protein that regulates the levels and localization of $\beta$-catenin during Wnt pathway activation and is involved in the genesis and progression of diseases like atrial septal defect, cryptorchidism, caudal duplication anomalies, breast cancer, non-small-cell lung cancer (NSCLC), hepatitis $B$ virus-related hepatocellular carcinoma (HCC), colorectal cancer, and gastrointestinal cancer [14-22].

AXIN1, the gene encoding AXIN1, is located within a $65 \mathrm{~kb}$ region on chromosome 16p. AXIN1 acts as a tumor suppressor, and mutations in this protein have been shown to play a significant role in carcinogenesis [23]. However, no link has been established so far between AXIN1 and BC. The aim of our study was to determine whether the polymorphic variants of AXIN1 contribute to BC susceptibility. We selected three tag single-nucleotide polymorphisms (SNPs) - rs12921862, rs1805105, and rs370681-and determined their prevalence in 316 unrelated BC patients and 419 healthy controls in a Chinese Han population.

\section{Material and Methods}

2.1. Participants' Clinical Characteristics and Follow-Up. We enrolled 316 patients with bladder cancer (mean age \pm SD: $63.76 \pm 12.14$ ) and 419 healthy controls (mean age \pm SD: $59.88 \pm 11.32$ ) from the West China Hospital of Sichuan University from 2007 to 2012. A case control study based on the hospital was approved by the hospital ethics committee, and informed consents were provided by all the participants. Follow-up data of participants were abstracted by telephone calls every 6 months for 5 years. Histopathological analysis was used to confirm the tumor tissues from resected specimens of patients, and the clinical characteristics are summarized in Table 1. This study excluded the control participants who had a personal or family history of BC or other severe diseases and the patients who had previous cancer or metastasized cancer from other origins as well as underwent radiotherapy or chemotherapy. All participants were genetically unrelated individuals of the Han population living in Sichuan province of China.
TABLE 1: Characteristics of the studied population.

\begin{tabular}{lcc}
\hline Characteristics & Patients & Controls \\
\hline Age at first diagnosis (mean \pm SD) & $63.76 \pm 12.14$ & $59.88 \pm 11.32$ \\
Sex & & \\
$\quad$ Male & $250(79.1 \%)$ & $225(53.7 \%)$ \\
$\quad$ Female & $66(20.9)$ & $194(46.3 \%)$ \\
Smoking status & & \\
Smoker & $163(51.6 \%)$ & $184(43.9 \%)$ \\
Nonsmoker & $153(48.4 \%)$ & $235(56.1 \%)$ \\
Tumor stage & & \\
MIBC & $149(47.2 \%)$ & \\
NMIBC & $167(52.8 \%)$ & \\
Tumor grade & & \\
High grade & $182(57.6 \%)$ & \\
Low grade & $134(42.4 \%)$ & \\
Clinical stage & & \\
I (Ta T1N0M0) & $156(53.1 \%)$ & \\
II (T2N0M0) & $84(28.6 \%)$ & \\
III (T3N0M0, T4aN0M0) & $34(11.6 \%)$ & \\
IV (T4bN0M0, TnNnM0, & $20(6.7 \%)$ & \\
TnNnMn, $n \geq 1)$ & & \\
\hline
\end{tabular}

2.2. AXIN1 Genotyping. The three tag SNPs were picked out according to data in the $\mathrm{CHB}$ population sample of the HapMap Project (Data Release 24/Phase II, NCBI build 36 assembly, dpSNPb126) using the algorithmTagger-pairwise Tagging from the international HapMap Project [24]. And the PCR primers were designed with software Primer 3 web version 4.1.0. (http://primer3.ut.ee/) [25] as shown in Table 2 (in this part, we followed the methods of Li et al. [26]).

DNA isolation kit from BioTeke (Peking, China) was used to extract each individual's genomic DNA from a $200 \mu \mathrm{L}$ EDTA-anticoagulated peripheral blood sample. Genotyping was performed using polymerase chain reaction-restriction fragment length polymorphism (PCR-RFLP). The DNA fragments containing the polymorphisms were amplified in a total volume of $10 \mu \mathrm{L}$, including $5 \mu \mathrm{L}$ of $2 \mathrm{x}$ power Taq PCR Master Mix (BioTeke, Peking, China), 2.7 pico mole of each primer, and $100 \mathrm{ng}$ genomic DNA for rs12921862 and rs1805105, and a total volume of $25 \mu \mathrm{L}$, including $2.5 \mu \mathrm{L}$ of Taq Buffer, $0.3 \mu \mathrm{L}$ Taq enzyme (BioTeke, Peking, China), $3 \mu \mathrm{L}$ DNTP, 2.7 pico mole of each primer, and $100 \mathrm{ng}$ genomic DNA for rs370681. The PCR conditions were $94^{\circ} \mathrm{C}$ for $4 \mathrm{~min}$, followed by 34 cycles of $30 \mathrm{~s}$ at $94^{\circ} \mathrm{C}, 30 \mathrm{~s}$ at $62^{\circ} \mathrm{C}$, $65^{\circ} \mathrm{C}$, or $66^{\circ} \mathrm{C}$, respectively, and $30 \mathrm{~s}$ at $72^{\circ} \mathrm{C}$, with a final elongation at $72^{\circ} \mathrm{C}$ for $10 \mathrm{~min}$ for three tag SNPs. Following the termination of PCR, the PCR products were digested by restriction enzyme (presented in Table 2). Finally, the digested fragments were separated by a $6 \%$ polyacrylamide gel and stained with $1.5 \mathrm{~g} / \mathrm{L}$ argent nitrate. Furthermore, the genotypes were confirmed by DNA sequencing analysis. About $10 \%$ of the samples were randomly selected to perform the repeated assays, and the results were $100 \%$ in agreement. 
TABLE 2: Primer sequences and reaction conditions for genotyping three tag SNPs in AXIN1 gene.

\begin{tabular}{|c|c|c|c|c|c|}
\hline SNPs & Primer sequence & Major/minor gene & $\begin{array}{c}\text { Annealing } \\
\text { temperature }\left({ }^{\circ} \mathrm{C}\right)\end{array}$ & Restriction enzyme & $\begin{array}{l}\text { Product size } \\
\text { (bp) }\end{array}$ \\
\hline \multirow{2}{*}{ rs12921862 } & F: 5'-CTCACGCCAGTGCCTCTACT-3' & \multirow{2}{*}{$\mathrm{C} / \mathrm{A}$} & \multirow{2}{*}{62} & \multirow{2}{*}{ ScrF I } & A: 216 \\
\hline & R: 5'-ATGCCATCCATGTGGAAACT-3' & & & & C: $110+106$ \\
\hline \multirow{2}{*}{ rs1805105 } & F: 5'-CTGGATACCTGCCGACCTTA-3' & \multirow{2}{*}{$\mathrm{T} / \mathrm{C}$} & \multirow{2}{*}{65} & \multirow{2}{*}{ Fok $I$} & C: 245 \\
\hline & R: 5'-ACCTTTCCCTGGCTTGTTCT-3' & & & & $\mathrm{T}: 186+59$ \\
\hline \multirow{2}{*}{ rs370681 } & F: $5^{\prime}$-GAGGCCTAAGCTCCAGGCACT-3' & \multirow{2}{*}{$\mathrm{C} / \mathrm{T}$} & \multirow{2}{*}{66} & \multirow{2}{*}{$B t s \alpha I$} & T: 166 \\
\hline & R: 5'-AAGGAAAGTGGGTTCTCCACCCA-3' & & & & C: $150+16$ \\
\hline
\end{tabular}

2.3. Statistical Analyses. SPSS for Windows software package version 20.0 (SPSS Inc., Chicago, IL, USA) was used to analysis the data. Genotypic association were provided by the SNPstats online analysis software including the codominant, dominant, recessive, and overdominant genetic models [27], and the Hardy-Weinberg equilibrium was evaluated by chisquared test. The level of significance was set at $P<0.05$. The effects of different genotypes and alleles were evaluated by odds ratio (OR) and respective $95 \%$ confidence intervals (95\% CI). Kaplan-Meier plots and the log-rank test were used to estimate the relationships of AXIN1 genotypes with patients' outcomes (recurrence and death). Considering age at first diagnosis, sex, smoking status, tumor grade, and clinical stage, Cox regression analysis model was used for multivariate survival analysis.

\section{Results}

3.1. AXIN1 SNP Frequencies and BC Susceptibility. The distributions of the three tag SNPs' genotypes and alleles in both case and control groups were in agreement with the Hardy-Weinberg equilibrium $(P>0.05)$. The differences in the genotypes and allele frequencies of AXIN1 SNPs between patients and controls are shown in Table 3. The frequency of the heterozygous genotype (CA) of rs12921862 was significantly higher among the patients compared to controls in the overdominant model $(33.5 \%$ vs. $8.8 \%, P<0.001$, $\mathrm{OR}=5.21,95 \% \mathrm{CI}=3.46-7.86)$. In the dominant model, a significantly increased $\mathrm{BC}$ risk was related to the CA/AA genotypes $(35.1 \%$ vs. $10.5 \%, P<0.001, \mathrm{OR}=4.61,95 \%$ $\mathrm{CI}=3.13-6.81)$. Compared to the CC/AA genotypes, the $\mathrm{CA}$ genotype was associated with a higher risk of $\mathrm{BC}$ in the codominant model $(P<0.001, \mathrm{OR}=5.24,95 \% \mathrm{CI}=3.47-$ 7.91). Similarly, a significant relationship was observed between the A allele-carrying patients and controls (18.4\% vs. $6.1 \%, P<0.001, \mathrm{OR}=3.45,95 \% \mathrm{CI}=2.44-5.00$ ). For rs 1805105, compared with TT carriers, TC/CC carriers were significantly higher in the dominant model $(46.5 \%$ vs. $39.1 \%$, $P=0.046, \mathrm{OR}=1.35,95 \% \mathrm{CI}=1.00-1.82)$. In the overdominant model, the TC genotype was significantly associated with increased $\mathrm{BC}$ risk compared to the TT/CC genotypes $(P=0.04, \mathrm{OR}=1.38,95 \% \mathrm{CI}=1.01-1.87)$. For rs370681, significant differences were found in the codominant (CC vs. CT vs. TT, $P=0.002, \mathrm{OR}_{\mathrm{CT}}=1.43,95 \%$ $\left.\mathrm{CI}_{\mathrm{CT}}=1.05-1.96, \mathrm{OR}_{\mathrm{TT}}=2.51,95 \% \mathrm{CI}_{\mathrm{TT}}=1.43-4.40\right)$, dominant (CC vs. CT/TT, $P=0.004$, $\mathrm{OR}=1.56,95 \% \mathrm{CI}=$ $1.15-2.10$ ), and recessive models (CC/CT vs. TT, $P=0.01$, $\mathrm{OR}=2.07,95 \% \mathrm{CI}=1.21-3.55)$ compared to healthy controls. Furthermore, the frequency of $\mathrm{T}$ allele was significantly higher in the $\mathrm{BC}$ patients compared to the healthy controls $(P=0.001, \mathrm{OR}=1.46,95 \% \mathrm{CI}=1.16-1.82)$.

3.2. Clinical Characteristics of the SNP Genotypes. To gain further insights into the association between the three tag SNPs and BC, the patients with different SNP genotypes were stratified (Table 4) according to age ( $\leq 64$ and $>64$ years old), sex (male and female), smoking status (smoker and nonsmoker), tumor stage (MIBC and NMIBC), tumor grade (high grade and low grade), recurrence status (recurrent and nonrecurrent), and metastasis status (metastatic and nonmetastatic). No significant difference was observed for any subgroups of the three tag SNPs except age and tumor grades. The CA/AA genotypes of rs 12921862 had a significantly higher percentage of patients older than 64 years compared with those under 64 years $(40.1 \%$ vs. $29.2 \%, P=0.04$, $\mathrm{OR}=1.64,95 \% \mathrm{CI}=1.01-2.70$ ), after adjusting for sex, smoking status, tumor stage, tumor grade, recurrence status, and metastasis status. For rs370681, after adjusting for these common risk factors, the $\mathrm{T}$ allele-carrying subgroup had a higher frequency of patients with high-grade tumors compared to those with low-grade tumors $(68 \%$ vs. $54 \%, P=0.04, \mathrm{OR}=1.85,95 \% \mathrm{CI}=1.04-3.23)$. In contrast, no significant relation was seen between the rs1805105 genotypes and the patients' characteristics after adjusting for common risk factors $(P>0.05)$.

3.3. Prognostic Value of the SNP Genotypes. The statistical association of the SNPs and overall survival of BC patients is summarized in Table 5. At the end of the follow-up in our study, 51 patients $(51 / 316 ; 16.1 \%)$ including 38 MIBC patients and 13 NMIBC patients had died and 93 patients (93/316; 29.4\%) including 46 MIBC patients and 47 NMIBC patients had relapsed. Kaplan-Meier survival analysis indicated worse prognosis of BC patients with the rs12921862 AA homozygote (log-rank: $P=0.003$, Figure 1). However, no significant relationship was observed between the overall survival and the other two SNPs (rs1805105 and rs370681) or between the three SNPs and recurrence-free survival.

Following the stratification of patients by tumor stage (MIBC and NMIBC), we conducted Cox univariate and 
TABLE 3: Distribution of SNPs in AXIN1 between patients and controls as well as their association with bladder cancer risk.

\begin{tabular}{|c|c|c|c|c|c|}
\hline \multirow{2}{*}{ Model } & \multirow{2}{*}{ Genotype } & \multirow{2}{*}{$\begin{array}{c}\text { Patients } \\
N(\%)\end{array}$} & \multirow{2}{*}{$\begin{array}{c}\text { Controls } \\
N(\%)\end{array}$} & \multicolumn{2}{|c|}{ Logistic regression } \\
\hline & & & & OR (95\% CI) & $P$ value \\
\hline \multicolumn{6}{|l|}{ rs12921862 } \\
\hline \multirow{3}{*}{ Codominant } & $\mathrm{CC}$ & 205 (64.9\%) & $375(89.5 \%)$ & 1.00 & \\
\hline & CA & $106(33.5 \%)$ & $37(8.8 \%)$ & $5.24(3.47-7.91)$ & $<0.001$ \\
\hline & AA & $5(1.6 \%)$ & $7(1.7 \%)$ & $1.31(0.41-4.17)$ & \\
\hline \multirow{2}{*}{ Dominant } & $\mathrm{CC}$ & 205 (64.9\%) & $375(89.5 \%)$ & 1.00 & \\
\hline & CA/AA & $111(35.1 \%)$ & $44(10.5 \%)$ & $4.61(3.13-6.81)$ & $<0.001$ \\
\hline \multirow{2}{*}{ Recessive } & $\mathrm{CC} / \mathrm{CA}$ & $311(98.4 \%)$ & $412(98.3 \%)$ & 1.00 & \\
\hline & AA & $5(1.6 \%)$ & $7(1.7 \%)$ & $0.95(0.30-3.01)$ & 0.93 \\
\hline \multirow{2}{*}{ Overdominant } & $\mathrm{CC} / \mathrm{AA}$ & $210(66.5 \%)$ & $382(91.2 \%)$ & 1.00 & \\
\hline & $\mathrm{CA}$ & $106(33.5 \%)$ & $37(8.8 \%)$ & $5.21(3.46-7.86)$ & $<0.001$ \\
\hline \multirow{2}{*}{ Allele } & $\mathrm{C}$ & $516(81.6 \%)$ & 787 (93.9\%) & 1.00 & \\
\hline & A & $116(18.4 \%)$ & $51(6.1 \%)$ & $3.45(2.44-5.00)$ & $<0.001$ \\
\hline \multicolumn{6}{|l|}{ rs1805105 } \\
\hline \multirow{3}{*}{ Codominant } & TT & $168(53.5 \%)$ & $255(60.9 \%)$ & 1.00 & \\
\hline & TC & $125(39.8 \%)$ & $136(32.5 \%)$ & $1.40(1.02-1.90)$ & 0.11 \\
\hline & $\mathrm{CC}$ & $21(6.7 \%)$ & $28(6.7 \%)$ & $1.14(0.63-2.07)$ & \\
\hline \multirow{2}{*}{ Dominant } & TT & $168(53.5 \%)$ & $255(60.9 \%)$ & 1.00 & \\
\hline & TC/CC & $146(46.5 \%)$ & $164(39.1 \%)$ & $1.35(1.00-1.82)$ & 0.046 \\
\hline \multirow{2}{*}{ Recessive } & TT/TC & $293(93.3 \%)$ & $391(93.3 \%)$ & 1.00 & \\
\hline & CC & $21(6.7 \%)$ & $28(6.7 \%)$ & $1.00(0.56-1.80)$ & 1 \\
\hline \multirow{2}{*}{ Overdominant } & TT/CC & $189(60.2 \%)$ & $283(67.5 \%)$ & 1.00 & \\
\hline & $\mathrm{TC}$ & $125(39.8 \%)$ & $136(32.5 \%)$ & $1.38(1.01-1.87)$ & 0.04 \\
\hline \multirow{2}{*}{ Allele } & $\mathrm{T}$ & $461(73.4 \%)$ & $646(77.1 \%)$ & 1.00 & \\
\hline & $\mathrm{C}$ & $167(26.6 \%)$ & $192(22.9 \%)$ & $1.22(0.96-1.55)$ & 0.11 \\
\hline \multicolumn{6}{|l|}{ rs370681 } \\
\hline \multirow{3}{*}{ Codominant } & $\mathrm{CC}$ & $114(37.9 \%)$ & $204(48.7 \%)$ & 1.00 & \\
\hline & CT & $152(50.5 \%)$ & $190(45.4 \%)$ & $1.43(1.05-1.96)$ & 0.002 \\
\hline & TT & $35(11.6 \%)$ & $25(6 \%)$ & $2.51(1.43-4.40)$ & \\
\hline \multirow{2}{*}{ Dominant } & $\mathrm{CC}$ & $114(37.9 \%)$ & $204(48.7 \%)$ & 1.00 & \\
\hline & $\mathrm{CT} / \mathrm{TT}$ & $187(62.1 \%)$ & $215(51.3 \%)$ & $1.56(1.15-2.10)$ & 0.004 \\
\hline \multirow{2}{*}{ Recessive } & $\mathrm{CC} / \mathrm{CT}$ & $266(88.4 \%)$ & $394(94 \%)$ & 1.00 & \\
\hline & $\mathrm{TT}$ & $35(11.6 \%)$ & $25(6 \%)$ & $2.07(1.21-3.55)$ & 0.01 \\
\hline \multirow{2}{*}{ Overdominant } & $\mathrm{CC} / \mathrm{TT}$ & $149(49.5 \%)$ & $229(54.6 \%)$ & 1.00 & \\
\hline & CT & $152(50.5 \%)$ & $190(45.4 \%)$ & $1.23(0.91-1.65)$ & 0.17 \\
\hline \multirow{2}{*}{ Allele } & $\mathrm{C}$ & $380(63.1 \%)$ & $598(71.4 \%)$ & 1.00 & \\
\hline & $\mathrm{T}$ & $222(36.9 \%)$ & $240(28.6 \%)$ & $1.46(1.16-1.82)$ & 0.001 \\
\hline
\end{tabular}

Boldfaced values indicate a significant difference at the $5 \%$ level. OR: odds ratio; CI: confidence interval.

multivariate analyses to determine the predictors of prognosis and survival in the different SNP genotypes (Table 5). Univariate analysis showed no significant association between tumor stage and overall survival in any genotype of the three SNPs in BC patients. However, the multivariate analysis showed a correlation between the CA/AA genotypes of AXIN1 rs12921862 and significantly higher overall survival rate $(P=0.03, \mathrm{OR}=0.1,95 \% \mathrm{CI}=0.01-0.84)$, compared to the CC genotype in NMIBC patients. KaplanMeier survival analysis reiterated that the CA/AA genotypes were associated with a better prognosis of NMIBC patients (log-rank: $P=0.03$, Figure 2) compared to the CC genotype.

\section{Discussion}

The Wnt signaling pathway plays a key role during embryonic development and tissue homeostasis, and its dysregulation is associated with various diseases including cancers $[7,8]$. The tumor suppressor function of AXIN1 and AXIN2 proteins in the Wnt signaling pathway has long been hypothesized, based largely on their roles in the $\beta$ catenin destruction complex. However, no study had directly linked AXIN1 with BC progression or the proliferation of BC cells. In the present study, we analyzed the potential relationship between different AXIN1 gene SNPs and BC risk in a 
TABLE 4: Distribution of SNPs in AXIN1 between patients' characteristics and their association with bladder cancer risk.

\begin{tabular}{|c|c|c|c|c|c|c|c|c|c|}
\hline \multirow{3}{*}{ Characteristics } & \multicolumn{3}{|c|}{ rs12921862 } & \multicolumn{3}{|c|}{ rs1805105 } & \multicolumn{3}{|c|}{ rs370681 } \\
\hline & \multicolumn{2}{|c|}{ Genotype } & \multirow[t]{2}{*}{$P$ value ${ }^{\mathrm{a}}$} & \multicolumn{2}{|c|}{ Genotype } & \multirow[t]{2}{*}{$P$ value $^{\mathrm{a}}$} & \multicolumn{2}{|c|}{ Genotype } & \multirow[t]{2}{*}{$P$ value } \\
\hline & $\mathrm{CC}$ & $\mathrm{CA} / \mathrm{AA}$ & & TT & TC/CC & & $\mathrm{CC}$ & $\mathrm{CT} / \mathrm{TT}$ & \\
\hline \multicolumn{10}{|l|}{ Age } \\
\hline$\leq 64$ years old & $102(70.8 \%)$ & $42(29.2 \%)$ & 0.04 & $85(59 \%)$ & $59(41 \%)$ & 0.10 & $56(40.3 \%)$ & 83 (59.7\%) & 0.83 \\
\hline$>64$ years old & 103 (59.9\%) & $69(40.1 \%)$ & & $83(48.8 \%)$ & 87 (51.2\%) & & $58(35.8 \%)$ & $104(64.2 \%)$ & \\
\hline \multicolumn{10}{|l|}{ Sex } \\
\hline Male & $164(65.9 \%)$ & $85(34.1 \%)$ & 0.77 & $128(51.6 \%)$ & $120(48.4 \%)$ & 0.47 & $88(37.5 \%)$ & $147(62.5 \%)$ & 0.96 \\
\hline Female & $41(61.2 \%)$ & $26(38.8 \%)$ & & $40(60.6 \%)$ & $26(39.4 \%)$ & & $26(39.4 \%)$ & $40(60.6 \%)$ & \\
\hline \multicolumn{10}{|l|}{ Smoke } \\
\hline Smoker & $111(68.1 \%)$ & $52(31.9 \%)$ & 0.4 & 80 (49.4\%) & $82(50.6 \%)$ & 0.25 & $58(37.7 \%)$ & $96(62.3 \%)$ & 0.90 \\
\hline Nonsmoker & $94(61.4 \%)$ & $59(38.6 \%)$ & & 88 (57.9\%) & $64(42.1 \%)$ & & $56(38.1 \%)$ & $91(61.9 \%)$ & \\
\hline \multicolumn{10}{|l|}{ Tumor stage } \\
\hline MIBC & $98(65.3 \%)$ & $52(34.7 \%)$ & 0.94 & 74 (49.7\%) & $75(50.3 \%)$ & 0.97 & $48(33.1 \%)$ & 97 (66.9\%) & 0.44 \\
\hline NMIBC & 107 (64.5\%) & $59(35.5 \%)$ & & $94(57 \%)$ & $71(43 \%)$ & & $66(42.3 \%)$ & $90(57.7 \%)$ & \\
\hline \multicolumn{10}{|l|}{ Tumor grade } \\
\hline High grade & $120(65.9 \%)$ & $62(34.1 \%)$ & 0.49 & 87 (48.3\%) & $93(51.7 \%)$ & 0.18 & $56(32 \%)$ & $119(68 \%)$ & 0.04 \\
\hline Low grade & $85(63.4 \%)$ & $49(36.6 \%)$ & & $81(60.5 \%)$ & $53(39.5 \%)$ & & $58(46 \%)$ & $68(54 \%)$ & \\
\hline \multicolumn{10}{|l|}{ Recurrence } \\
\hline Recurrent & $59(63.4 \%)$ & $34(36.6 \%)$ & 0.84 & $50(53.8 \%)$ & $43(46.2 \%)$ & 0.97 & $32(36 \%)$ & $57(64 \%)$ & 0.40 \\
\hline Nonrecurrent & $146(65.5 \%)$ & 77 (34.5\%) & & $118(53.4 \%)$ & $103(46.6 \%)$ & & $82(38.7 \%)$ & $130(61.3 \%)$ & \\
\hline \multicolumn{10}{|l|}{ Metastasis } \\
\hline Metastatic & $31(62 \%)$ & $19(38 \%)$ & 0.68 & $24(49 \%)$ & $25(51 \%)$ & 0.70 & $19(40.4 \%)$ & $28(59.6 \%)$ & 0.07 \\
\hline Nonmetastatic & $174(65.4 \%)$ & $92(34.6 \%)$ & & $144(54.3 \%)$ & $121(45.7 \%)$ & & $94(37.1 \%)$ & $159(62.9 \%)$ & \\
\hline
\end{tabular}

Italic values indicate a significant difference at the $5 \%$ level. ${ }^{a}$ Adjusted by age, sex, smoking status, tumor stage, tumor grade, recurrence status, and metastasis status.

Chinese Han population. Despite extensive linkage disequilibrium in the human genome, a tag SNP could represent most of the haplotypes in a specific region. Our data revealed that the three tag SNPs were associated with an increased risk of $\mathrm{BC}$, which corresponded to the previous observations that activating or inhibiting Wnt signaling accelerates or suppresses, respectively, the invasion and migration of $\mathrm{BC}$ cells as well as the carcinogenesis of $\mathrm{BC}$ tumor $[12,13]$. We calculated the statistical power using Power and Sample Size Calculations for Windows software package version 3.0 [28], and it showed that our data had more than $80 \%$ power to detect the relationship between AXIN1 SNPs and BC risk.

Tumor suppressor protein AXIN1 deregulates $\beta$-catenin and mitosis to weaken the $\mathrm{ER}^{+}$and $\mathrm{ER}^{-}$breast cancer $[14,19]$. It interacts with $\beta$-catenin and regulates its localization, along with that of Wnt-dependent downstream targets like cyclin D1, fra-1, c-myc, and c-jun [18, 29]. One previous study showed that poorly differentiated NSCLC had low levels of AXIN1 relating a high expression of nuclear $\beta$-catenin, which was reversed in the well- or moderately differentiated tumors [30]. The oncogenic effects of AXIN monoallele mutations that lead to $\beta$-catenin stabilization result from the dominant negative activity [31]. Compared to the CC homozygote of the rs12921862 SNP, the CA/AA genotypes were associated with a significantly higher overall survival rate and implicated in a better prognosis of the $\mathrm{BC}$ patients, which might indicate a potential dominant negative protective effect of the latter in NMIBC patients. Furthermore, these results are consistent with the better prognosis of NMIBC patients compared to the MIBC.

Tumor stratification is used to grade cancers according to their malignancy: high-grade tumor classification normally represents a worse prognosis compared to that of the low grade. Studies have shown that the AXIN1 rs1805105 polymorphism is correlated with the early tumor stages (I and II, modified by the Union for International Cancer Control) and the small tumor size (under $5 \mathrm{~cm}$ ) of HCC [32] but increases the risk of developing advance-stage renal carcinogenesis (RCC III and IV) [33]. However, we found no significant association between the rs1805105 genotypes and tumor grade in our study, although the $\mathrm{C}$ allele carriers had a higher (albeit nonsignificant: $51.7 \%$ vs. $39.5 \%$ ) frequency of patients with high-grade tumor compared to those with low-grade tumors. This indicated a nonsignificant tendency that the $\mathrm{C}$ allele may be correlated with the potential risk of developing higher-grade tumors among $\mathrm{BC}$ patients. Interestingly, the frequency of $\mathrm{T}$ allele carriers of the rs370681 SNP with high-grade tumors was significantly elevated than that with low-grade tumors, which indicated an association of this SNP with severe BC. These results are consistent with previous studies on HCC and RCC.

Bladder cancer is an older-susceptible cancer although the average age of the afflicted has decreased over these years. In this study also, we found that the CA/AA carriers of 
TABle 5: Association between SNPs in AXIN1 and overall survival for patients with MIBC or NMIBC.

\begin{tabular}{|c|c|c|c|c|c|c|}
\hline \multirow{2}{*}{ SNP/genotype } & \multicolumn{3}{|c|}{ MIBC } & \multicolumn{3}{|c|}{ NMIBC } \\
\hline & Alive/dead & $\operatorname{HR}(95 \% \mathrm{CI})^{\mathrm{a}}$ & $P$ value $^{\mathrm{a}}$ & Alive/dead & $\operatorname{HR}(95 \% \mathrm{CI})^{\mathrm{a}}$ & $P$ value $^{\mathrm{a}}$ \\
\hline \multicolumn{7}{|l|}{ rs12921862 } \\
\hline $\mathrm{CC}$ & $75 / 22$ & & & $96 / 12$ & & \\
\hline $\mathrm{CA}$ & $34 / 13$ & & & $58 / 1$ & & \\
\hline $\mathrm{AA}$ & $2 / 3$ & & & $0 / 0$ & & \\
\hline Codominant & & $1.41(0.83-2.42)$ & 0.21 & & $0.10(0.01-0.84)$ & 0.03 \\
\hline Dominant & & $1.45(0.74-2.88)$ & 0.28 & & $0.10(0.01-0.84)$ & 0.03 \\
\hline Recessive & & $1.94(0.53-6.89)$ & 0.32 & & NA & NA \\
\hline \multicolumn{7}{|l|}{ rs1805105 } \\
\hline $\mathrm{TT}$ & $54 / 19$ & & & $89 / 6$ & & \\
\hline $\mathrm{TC}$ & $46 / 16$ & & & $56 / 7$ & & \\
\hline $\mathrm{CC}$ & $11 / 2$ & & & $8 / 0$ & & \\
\hline Codominant & & $1.40(0.71-2.76)$ & 0.34 & & $0.97(0.34-2.72)$ & 0.95 \\
\hline Dominant & & $1.21(0.58-2.54)$ & 0.62 & & $1.12(0.35-3.62)$ & 0.85 \\
\hline Recessive & & $4.28(0.88-20.88)$ & 0.07 & & $0.00(0.00-\mathrm{NA})$ & 0.99 \\
\hline \multicolumn{7}{|l|}{ rs370681 } \\
\hline $\mathrm{CC}$ & $37 / 10$ & & & $60 / 7$ & & \\
\hline $\mathrm{CT}$ & $55 / 23$ & & & $69 / 5$ & & \\
\hline $\mathrm{TT}$ & $16 / 3$ & & & $16 / 0$ & & \\
\hline Codominant & & $1.30(0.72-2.34)$ & 0.38 & & $0.54(0.19-1.54)$ & 0.25 \\
\hline Dominant & & $1.76(0.76-4.06)$ & 0.18 & & $0.60(0.18-1.97)$ & 0.40 \\
\hline Recessive & & $0.82(0.21-3.17)$ & 0.77 & & $0.00(0.00-\mathrm{NA})$ & 0.99 \\
\hline
\end{tabular}

Italic values indicate a significant difference at the $5 \%$ level. ${ }^{a}$ Adjusted by age, sex, smoking status, tumor stage, tumor grade, recurrence status, and metastasis status. HR: hazard ratio; CI: confidence interval.

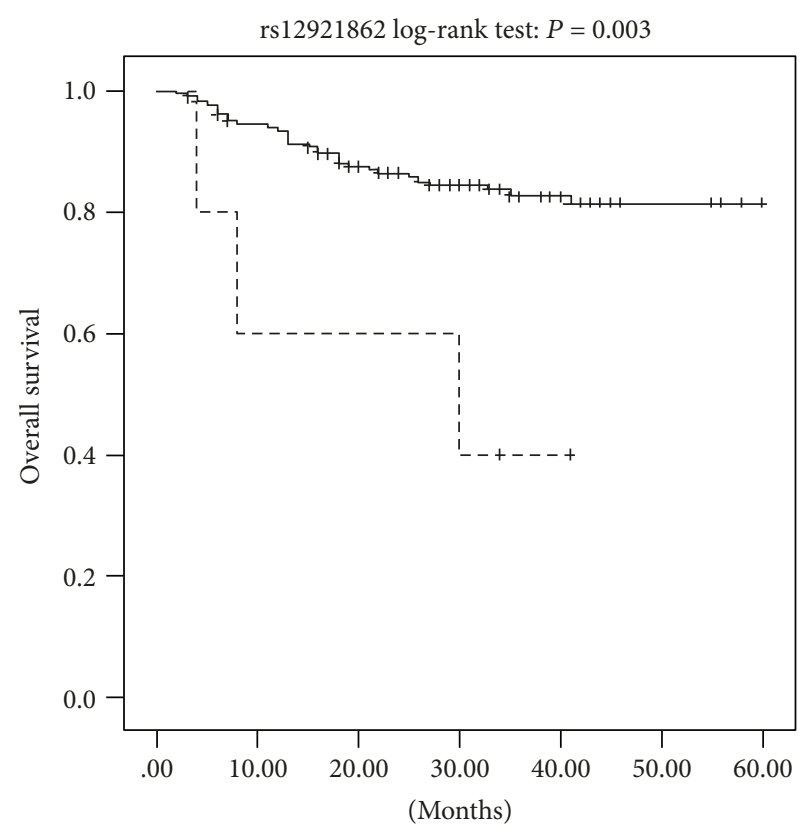

$$
\begin{array}{ll}
\begin{array}{l}
\text { Genotype } \\
\neg \mathrm{CC} / \mathrm{CA}
\end{array} & + \text { CC/CA-censored } \\
--\mathrm{AA} & + \text { AA-censored }
\end{array}
$$

Figure 1: Kaplan-Meier survival curves for the recessive model of AXIN1 rs12921862 polymorphism in BC patients.

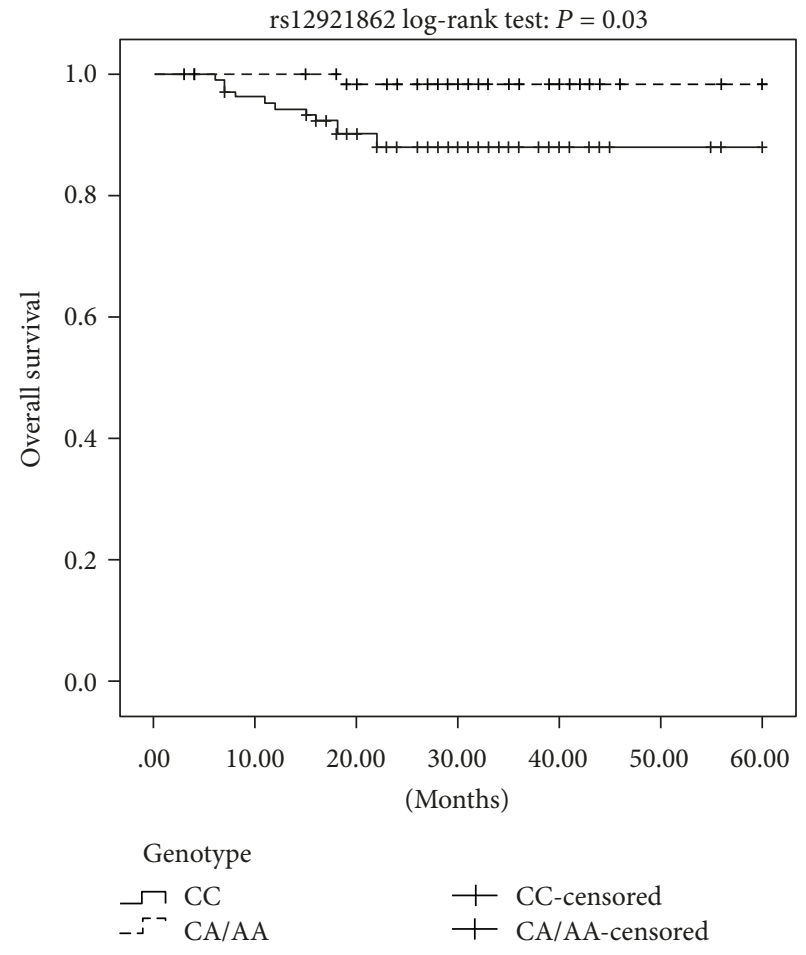

FIGURE 2: Kaplan-Meier survival curves for the dominant model of AXIN1 rs12921862 polymorphism in NMIBC patients. 
rs12921862 SNP had significantly higher frequencies of patients older than 64 years compared to those under 64 years, indicating that the A allele could be one of the risk factors of increased BC susceptibility in the older population.

A correlation between reduced AXIN1 expression and tumor progression has been reported in esophageal squamous cell carcinoma, and several mutations and polymorphisms of AXIN1 have been found in squamous cell tumors and cell lines $[23,34,35]$. Several recent studies have detected AXIN1 gene sequence variations in subsets of ovarian endometrial adenocarcinomas and advanced prostate cancer, indicating new potentially relevant $A X I N 1$ mutations $[23,36]$. Furthermore, Chimge et al. found that AXIN1 could be a potential target for the management of $\mathrm{ER}^{+}$breast cancer [14]. Our study demonstrated the association of certain AXIN1 gene polymorphisms with higher BC susceptibility and higher overall survival rate of NMIBC patients. Our results provide a new insight into potential predictive factors of $\mathrm{BC}$ progression and prognosis.

\section{Conclusions}

In conclusion, AXIN1 SNPs are potential risk factors of BC susceptibility, rs370681 is associated with a severe form of $\mathrm{BC}$, and rs12921862 is a significant forecast factor to BC prognosis. However, our study has several limitations. We did not analyze the expression levels of AXIN1 in the patients' sera and tumor tissues. Also, we only studied a cohort of southwest China, whereas different ethnic populations differ in the types and frequencies of genetic polymorphisms. Therefore, further studies on larger and more diverse cohorts are needed to validate AXIN1 SNPs as reliable markers to predict the progression and prognosis of bladder cancer.

\section{Data Availability}

The data used to support the findings of this study are currently under embargo while the research findings are commercialized. Requests for data, 6 months after publication of this article, will be considered by the corresponding authors.

\section{Conflicts of Interest}

The authors declare that they have no conflict of interest.

\section{Authors' Contributions}

Qin Li and Peng Zhang contributed equally to this work.

\section{Acknowledgments}

This work was supported by the National Natural Science Foundation of China (no. 81670346 and no. 81172440), the Applied Basic Research Programs of Science and Technology Commission Foundation of Sichuan Province (no. 2016SZ0013), the International Cooperation Project of the Science and Technology Department of Sichuan Province (no. 2018HH0014), and Science and Technology Major
Project of Sichuan Province (no. 2017SZDZX0013). In addition, we especially wish to thank Yan $\mathrm{Pu}$, who provided part of the important data of participants.

\section{References}

[1] J. Ferlay, I. SoerjomataramI, M. Ervik et al., Globocan 2012 v1.0, Cancer Incidence and Mortality Worldwide: Iarc CancerBase no. 11, International agency for research on cancer, http://globocan.Iarc.Fr.

[2] R. L. Siegel, K. D. Miller, and A. Jemal, "Cancer statistics, 2015," CA: A Cancer Journal for Clinicians, vol. 65, no. 1, pp. 5-29, 2015.

[3] M. Babjuk, A. Böhle, M. Burger et al., "Eau guidelines on nonmuscle-invasive urothelial carcinoma of the bladder: update 2016," European Urology, vol. 71, no. 3, pp. 447-461, 2017.

[4] M. Burger, J. W. F. Catto, G. Dalbagni et al., "Epidemiology and risk factors of urothelial bladder cancer," European Urology, vol. 63, no. 2, pp. 234-241, 2013.

[5] N. D. Freedman, D. T. Silverman, A. R. Hollenbeck, A. Schatzkin, and C. C. Abnet, "Association between smoking and risk of bladder cancer among men and women," JAMA, vol. 306, no. 7, pp. 737-745, 2011.

[6] J. Gu, D. Liang, Y. Wang, C. Lu, and X. Wu, "Effects of n-acetyl transferase 1 and 2 polymorphisms on bladder cancer risk in caucasians," Mutation Research, vol. 581, no. 1-2, pp. 97104, 2005.

[7] R. Nusse and H. Clevers, "Wnt/ $\beta$-catenin signaling, disease, and emerging therapeutic modalities," Cell, vol. 169, no. 6, pp. 985-999, 2017.

[8] C. Y. Logan and R. Nusse, "The wnt signaling pathway in development and disease," Annual Review of Cell and Developmental Biology, vol. 20, no. 1, pp. 781-810, 2004.

[9] S. Urakami, H. Shiina, H. Enokida et al., "Combination analysis of hypermethylated wnt-antagonist family genes as a novel epigenetic biomarker panel for bladder cancer detection," Clinical Cancer Research, vol. 12, no. 7, pp. 2109-2116, 2006.

[10] K. Shin, J. Lee, N. Guo et al., "Hedgehog/wnt feedback supports regenerative proliferation of epithelial stem cells in bladder," Nature, vol. 472, no. 7341, pp. 110-114, 2011.

[11] S. Urakami, H. Shiina, H. Enokida et al., "Epigenetic inactivation of wnt inhibitory factor- 1 plays an important role in bladder cancer through aberrant canonical wnt $\beta$-catenin signaling pathway," Clinical Cancer Research, vol. 12, no. 2, pp. 383-391, 2006.

[12] X. W. Mao, J. Q. Xiao, Z. Y. Li, Y. C. Zheng, and N. Zhang, "Effects of microrna-135a on the epithelial-mesenchymal transition, migration and invasion of bladder cancer cells by targeting gsk $3 \beta$ through the wnt $/ \beta$-catenin signaling pathway," Experimental and Molecular Medicine, vol. 50, no. 1, article e429, 2018.

[13] Y. Tang, A. R. Simoneau, W. X. Liao et al., "Wif1, a wnt pathway inhibitor, regulates skp2 and c-myc expression leading to g1 arrest and growth inhibition of human invasive urinary bladder cancer cells," Molecular Cancer Therapeutics, vol. 8, no. 2, pp. 458-468, 2009.

[14] N. O. Chimge, G. H. Little, S. K. Baniwal et al., "Runx1 prevents oestrogen-mediated axin 1 suppression and $\beta$-catenin activation in er-positive breast cancer," Nature Communications, vol. 7, no. 1, article 10751, 2016. 
[15] L. Fang, J. Cai, B. Chen et al., "Aberrantly expressed mir$582-3 \mathrm{p}$ maintains lung cancer stem cell-like traits by activating wnt/ $\beta$-catenin signalling," Nature Communications, vol. 6, no. 1, article 8640, 2015.

[16] S. Abitbol, R. Dahmani, C. Coulouarn et al., "Axin deficiency in human and mouse hepatocytes induces hepatocellular carcinoma in the absence of $\beta$-catenin activation," Journal of Hepatology, vol. 68, no. 6, pp. 1203-1213, 2018.

[17] Y. Lu, S. Xie, W. Zhang et al., "Twal/gid8 is a $\beta$-catenin nuclear retention factor in wnt signaling and colorectal tumorigenesis," Cell Research, vol. 27, no. 12, pp. 1422-1440, 2017.

[18] S. M. Mazzoni and E. R. Fearon, "Axin1 and axin2 variants in gastrointestinal cancers," Cancer Letters, vol. 355, no. 1, pp. 1$8,2014$.

[19] N. O. Chimge, S. Ahmed-Alnassar, and B. Frenkel, "Relationship between runx 1 and axin 1 in er-negative versus er-positive breast cancer," Cell Cycle, vol. 16, no. 4, pp. 312-318, 2017.

[20] N. A. Oates, J. van Vliet, D. L. Duffy et al., "Increased DNA methylation at the axin1 gene in a monozygotic twin from a pair discordant for a caudal duplication anomaly," American Journal of Human Genetics, vol. 79, no. 1, pp. 155-162, 2006.

[21] Y. Pu, P. Chen, B. Zhou et al., "Association between polymorphisms in AXIN1 gene and atrial septal defect," Biomarkers, vol. 19, no. 8, pp. 674-678, 2014.

[22] B. Zhou, T. Tang, P. Chen et al., "The variations in the axin1 gene and susceptibility to cryptorchidism," Journal of Pediatric Urology, vol. 11, no. 3, pp. 132.e1-132.e5, 2015.

[23] S. Salahshor and J. R. Woodgett, "The links between axin and carcinogenesis," Journal of Clinical Pathology, vol. 58, no. 3, pp. 225-236, 2005.

[24] "Ncbi retiring hapmap resource," http://www.Hapmap.Org/ index.Html.Zh.

[25] A. Untergasser, I. Cutcutache, T. Koressaar et al., "Primer3new capabilities and interfaces," Nucleic Acids Research, vol. 40, no. 15, article E115, 2012.

[26] Q. Li, T. Tang, P. Zhang et al., "Correlation of il-31 gene polymorphisms with susceptibility and clinical recurrence of bladder cancer," Familial Cancer, vol. 17, no. 4, pp. 577$585,2018$.

[27] X. Sole, E. Guino, J. Valls, R. Iniesta, and V. Moreno, "Snpstats: a web tool for the analysis of association studies," Bioinformatics, vol. 22, no. 15, pp. 1928-1929, 2006.

[28] W. D. Dupont and W. D. Plummer Jr., "Power and sample size calculations. A review and computer program," Controlled Clinical Trials, vol. 11, no. 2, pp. 116-128, 1990.

[29] A. Kikuchi, "Modulation of wnt signaling by axin and axil," Cytokine and Growth Factor Reviews, vol. 10, no. 3-4, pp. 255-265, 1999.

[30] H. T. Xu, L. Wang, D. Lin et al., “Abnormal $\beta$-catenin and reduced axin expression are associated with poor differentiation and progression in non-small cell lung cancer," American Journal of Clinical Pathology, vol. 125, no. 4, pp. 534541,2006

[31] J. Behrens, B. A. Jerchow, M. Würtele et al., "Functional interaction of an axin homolog, conductin, with $\beta$-catenin, apc, and gsk3 $\beta$," Science, vol. 280, no. 5363, pp. 596-599, 1998.

[32] S. S. Kim, H. J. Cho, H. Y. Lee et al., "Genetic polymorphisms in the wnt/ $\beta$-catenin pathway genes as predictors of tumor development and survival in patients with hepatitis B virusassociated hepatocellular carcinoma," Clinical Biochemistry, vol. 49, no. 10-11, pp. 792-801, 2016.
[33] Y. Pu, X. Mi, P. Chen et al., "Genetic association of polymorphisms in AXIN1 gene with clear cell renal cell carcinoma in a chinese population," Biomarkers in Medicine, vol. 11, no. 11, pp. 947-955, 2017.

[34] M. Nakajima, M. Fukuchi, T. Miyazaki, N. Masuda, H. Kato, and H. Kuwano, "Reduced expression of axin correlates with tumour progression of oesophageal squamous cell carcinoma," British Journal of Cancer, vol. 88, no. 11, pp. 1734-1739, 2003.

[35] J. de Castro, C. Gamallo, J. Palacios et al., “ $\beta$-catenin expression pattern in primary oesophageal squamous cell carcinoma. Relationship with clinicopathologic features and clinical outcome," Virchows Archiv, vol. 437, no. 6, pp. 599-604, 2000.

[36] G. W. Yardy, D. C. Bicknell, J. L. Wilding et al., "Mutations in the axin 1 gene in advanced prostate cancer," European Urology, vol. 56, no. 3, pp. 486-494, 2009. 


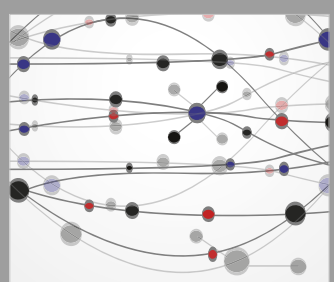

The Scientific World Journal
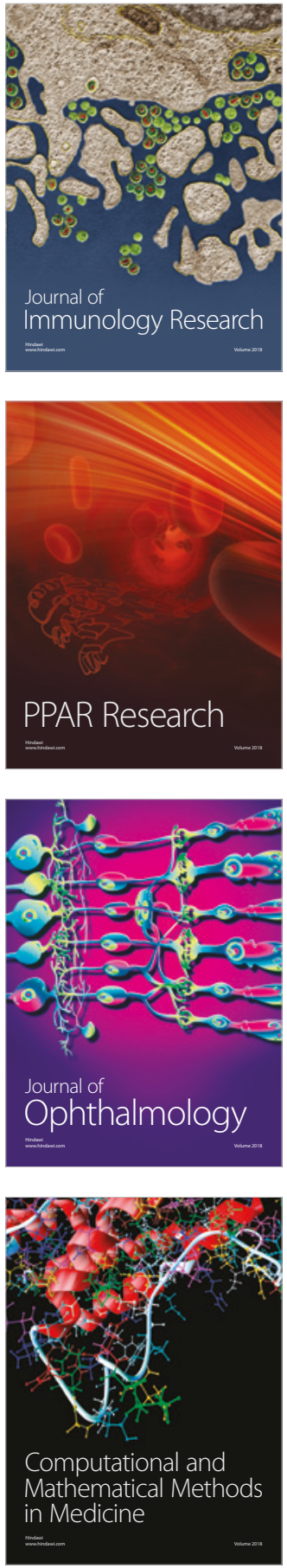

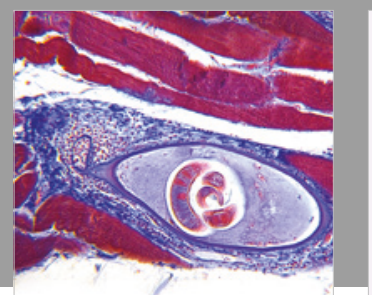

Gastroenterology Research and Practice

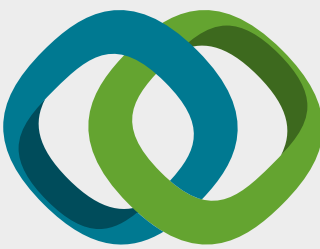

\section{Hindawi}

Submit your manuscripts at

www.hindawi.com
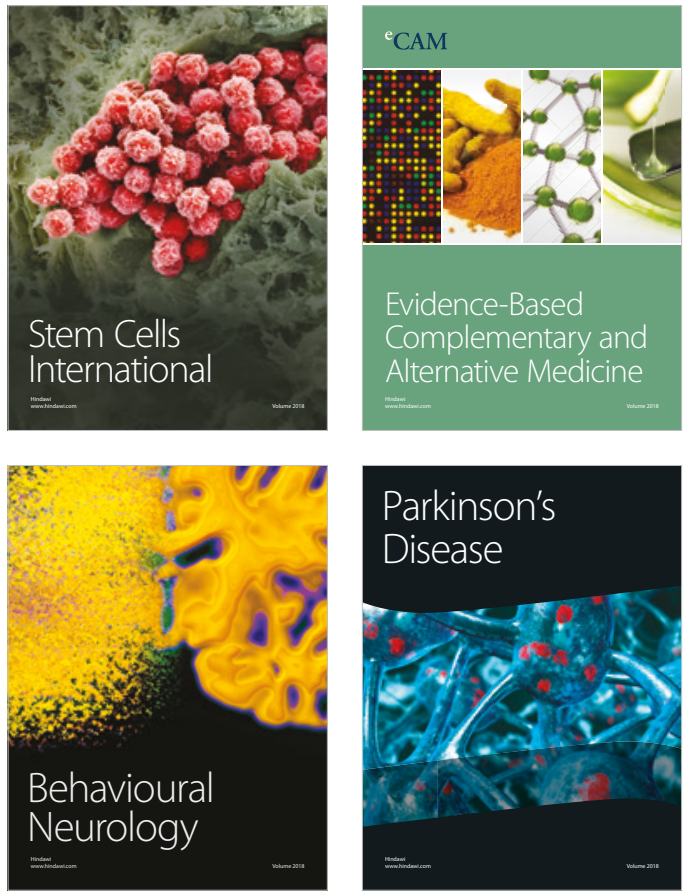

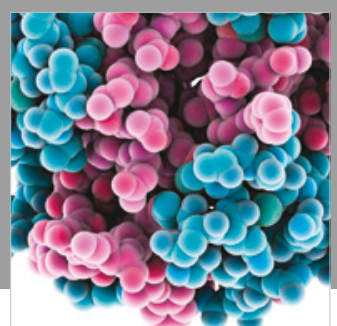

ournal of

Diabetes Research

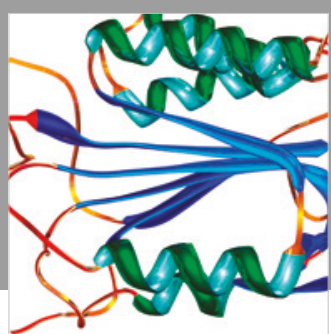

Disease Markers
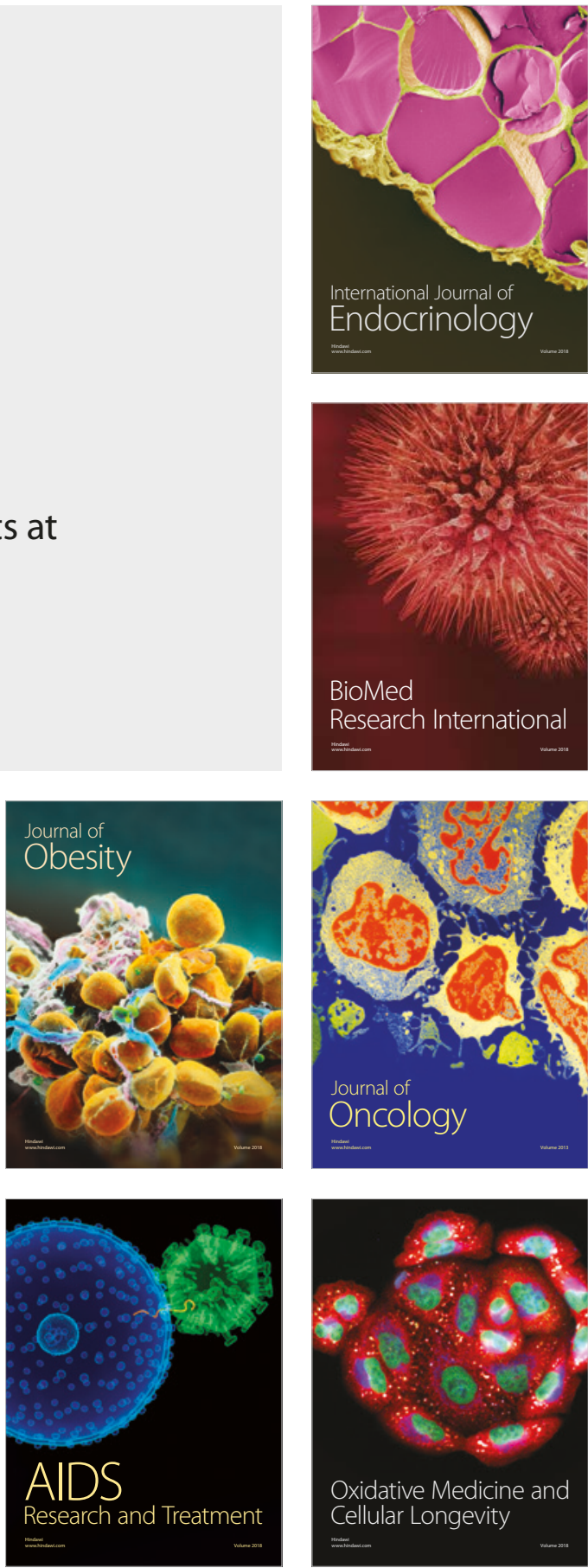